Supporting information

\title{
Strong Coupling and Slow Relaxation of the Magnetization for an Air-Stable [Co4] Square with Both Tetrazine Radicals and Azido Bridges
}

\author{
Zhilin Guo, ${ }^{\dagger,}$ Yi-Fei Deng, ${ }^{\dagger}, *$ Zoe Pikramenou, ${ }^{\ddagger}$ Kim R. Dunbar,${ }^{\S} *$ and Yuan-Zhu Zhang ${ }^{\dagger, *}$ \\ † Department of Chemistry, Southern University of Science and Technology, Shenzhen 518055, China. \\ * School of Chemistry, The University of Birmingham, Edgbaston B15 2TT, United Kingdom. \\ ${ }^{\S}$ Department of Chemistry, Texas A \& M University, College Station, Texas 77842, United States.
}

Email: dengyf@sustech.edu.cn; dunbar@chem.tamu.edu; zhangyz@sustech.edu.cn 


\section{EXPERIMENTAL SECTION}

Materials and Syntheses. All syntheses were carried out under an argon atmosphere using a glove box. The starting materials are from commercially available sources and were used as received. Acetonitrile and dichloromethane were purified using a solvent purification system and stored over molecular sieves in the glove box. Anhydrous methanol was purchased from Energy Chem and stored over molecular sieves in a glove box.

Caution. Although no such issues were observed during the present work, azido and perchlorate salts are potentially explosive and should be handled with extreme caution.

Synthesis of $\left[\mathbf{Z n}_{4}\left(\mathbf{b p z t z}^{-}\right)_{\mathbf{4}}\left(\mathbf{N}_{\mathbf{3}}\right)_{4}\right] \mathbf{( 1 )}$ : $\left[\mathrm{Zn}\left(\mathrm{H}_{2} \mathrm{O}\right)_{6}\right]\left[\mathrm{ClO}_{4}\right]_{2}(37.2 \mathrm{mg}, 0.1 \mathrm{mmol})$ and $\mathrm{L}$-ascorbic acid $(26.5 \mathrm{mg}, 0.15 \mathrm{mmol})$ were dissolved in $3 \mathrm{~mL}$ acetonitrile and $1 \mathrm{~mL}$ methanol, to which bpztz (27.2 mg, $0.1 \mathrm{mmol})$ in $4 \mathrm{~mL}$ dichloromethane was added, followed by $\mathrm{NaN}_{3}(9.1 \mathrm{mg}, 0.14 \mathrm{mmol})$ in $4 \mathrm{~mL}$ methanol. The resulting brown solution was slowly evaporated at room temperature for three days which yielded black block crystals of $\mathbf{1}$. The product was collected by filtration and washed with acetonitrile. Yield $9.6 \mathrm{mg}$ (26 \% based on bpztz). Anal. Calc. for $\mathrm{C}_{48} \mathrm{H}_{56} \mathrm{Zn}_{4} \mathrm{~N}_{44} \mathrm{C}, 38.16 \%$; $3.74 \%$; N, $40.79 \%$. Found C, $38.34 \%$; H, $3.88 \%$; N, $40.29 \%$. Selected IR data (cm-1): 2361 (m), 2338 (w), 2074 (s), 1573 (m), 1477 (m), 1408 (s), 1320 (m), $1294(\mathrm{~m}), 1121(\mathrm{~m}), 1063(\mathrm{~m}), 1043(\mathrm{~m}), 985$ (m).

Synthesis of [Co4 $\left.\left(\text { bpztz }^{-}\right)_{4}\left(\mathbf{N}_{3}\right)_{4}\right]$ (2): Black block crystals of 2 were obtained by following a similar procedure to that of 1 with the starting material $\left[\mathrm{Co}\left(\mathrm{H}_{2} \mathrm{O}\right)_{6}\right]\left[\mathrm{ClO}_{4}\right]_{2}(36.8 \mathrm{mg}, 0.1 \mathrm{mmol})$. Yield $13.4 \mathrm{mg}$ (36 \% based on bpztz). Anal. Calc. for $\mathrm{C}_{48} \mathrm{H}_{56} \mathrm{Co}_{4} \mathrm{~N}_{44} \mathrm{C}, 38.82 \% ; \mathrm{H}, 3.80 \%$; N, $41.50 \%$. Found C, $38.65 \% ; \mathrm{H}, 3.49 \%$; N, $41.25 \%$. Selected IR data $\left(\mathrm{cm}^{-1}\right): 2363$ (m), 2338 (w), 2053 (s), 1571 (m), 1478 (m), 1411 (s), 1323 (m), 1283 (m), 1125 (m), 1065 (m), 1041 (m), 987 (m).

Physical Measurements. X-ray data for 1 and 2 were collected on a Bruker APEX-II CCD diffractometer using graphite monochromated $\mathrm{Cu} \mathrm{K} \alpha$ radiation $(\lambda=1.54178 \AA$ ). The structure was solved with the ShelXT structure solution program using Intrinsic Phasing and refined in the ShelXL refinement package using Least Squares minimization. ${ }^{1}$ Hydrogen atoms were added at calculated positions and refined using a riding model. Weighted R factors (wR) and the goodness-of-fit (S) values are based on $\mathrm{F}^{2}$; conventional $\mathrm{R}$ factors $(\mathrm{R})$ are based on $\mathrm{F}$, with $\mathrm{F}$ set to zero for negative $\mathrm{F}^{2}$. Powder X-ray Diffraction (PXRD) measurements were recorded on a Rigaku Smartlab X-ray diffractometer. FT-IR spectra were recorded in the range 600-4000 $\mathrm{cm}^{-1}$ on a Bruker tensor II spectrophotometer. Elemental analyses $(\mathrm{C}, \mathrm{H}, \mathrm{N})$ were measured by a vario EL cube CHNOS Elemental Analyzer Elementar Analysensysteme GmbH. UV-vis absorption spectra were recorded on an Agilent Cary 5000 UV-vis spectrophotometer. Solid state reflectance spectra were recorded on the same instrument with a corresponding integrating sphere from Agilent. Cyclic voltammetry (CV) measurements were carried out on a CHI760E electrochemical workstation (Chenhua Co. Ltd., China) equipped with a Pt working electrode. $\mathrm{Ag} / \mathrm{AgCl}$ was used as the reference electrode. The measurements were performed under nitrogen atmosphere in dichloromethane $\left(\mathrm{CH}_{2} \mathrm{Cl}_{2}\right)$ solution with a $0.4 \mathrm{mM}$ concentration of the complex in the presence of $0.1 \mathrm{M}\left[\mathrm{n}-\mathrm{Bu}_{4} \mathrm{~N}\right]\left[\mathrm{PF}_{6}\right]$ as the supporting electrolyte at a scan rate of $0.1 \mathrm{~V} \cdot \mathrm{s}^{-1}$. The specific heat measurements were conducted using a PPMS DynaCool-9T system. Magnetic measurements were carried out on a SQUID MPMS3 magnetometer. Diamagnetic corrections were calculated from Pascal constants and applied to all the constituent atoms and sample holder. ${ }^{2}$

Table S1. Crystallographic data of $\mathbf{1}$ and $\mathbf{2 .}$

\begin{tabular}{|c|c|c|}
\hline & 1 & 2 \\
\hline Empirical formula & $\mathrm{C}_{48} \mathrm{H}_{56} \mathrm{~N}_{44} \mathrm{Zn}_{4}$ & $\mathrm{C}_{48} \mathrm{H}_{56} \mathrm{Co}_{4} \mathrm{~N}_{44}$ \\
\hline Formula weight & 1510.84 & 1485.08 \\
\hline Temperature/K & $150(2)$ & $100(2)$ \\
\hline Crystal system & tetragonal & tetragonal \\
\hline Space group & $\mathrm{I}_{1} / \mathrm{acd}$ & $\mathrm{I} 4_{1} / \mathrm{acd}$ \\
\hline $\mathrm{a} / \AA ̊$ & $17.3851(8)$ & $17.3642(5)$ \\
\hline $\mathrm{b} / \AA$ & $17.3851(8)$ & $17.3642(5)$ \\
\hline $\mathrm{c} / \AA$ & $42.117(2)$ & $41.7879(12)$ \\
\hline
\end{tabular}




\begin{tabular}{|c|c|c|}
\hline$\overline{\alpha /{ }^{\circ}}$ & 90 & 90 \\
\hline$\beta /^{\circ}$ & 90 & 90 \\
\hline$\gamma /{ }^{\circ}$ & 90 & 90 \\
\hline Volume/ $\AA^{3}$ & $12729.6(13)$ & $12599.7(8)$ \\
\hline $\mathrm{Z}$ & 8 & 8 \\
\hline$\rho_{\text {calc }} \mathrm{g} / \mathrm{cm}^{3}$ & 1.577 & 1.566 \\
\hline$\mu / \mathrm{mm}^{-1}$ & 2.333 & 8.734 \\
\hline $\mathrm{F}(000)$ & 6176 & 6080 \\
\hline Crystal size $/ \mathrm{mm}^{3}$ & $0.2 \times 0.2 \times 0.15$ & $0.21 \times 0.18 \times 0.07$ \\
\hline Radiation & $\mathrm{CuK} \alpha(\lambda=1.54178)$ & $\mathrm{CuK} \alpha(\lambda=1.54178)$ \\
\hline $2 \theta$ range for data collection $/^{\circ}$ & 11.01 to 136.96 & 8.35 to 136.81 \\
\hline Reflections collected & 30639 & 27656 \\
\hline Independent reflections & $2930\left[R_{\text {int }}=0.0487\right]$ & $2898\left[\mathrm{R}_{\mathrm{int}}=0.0754\right]$ \\
\hline Goodness-of-fit on $\mathrm{F}^{2}$ & 1.077 & 1.037 \\
\hline completeness & $99.8 \%$ & $99.9 \%$ \\
\hline Final $R$ indexes $[I>2 \sigma(I)]^{a, b}$ & $\mathrm{R}_{1}=0.0417, \mathrm{w} \mathrm{R}_{2}=0.1007$ & $\mathrm{R}_{1}=0.0379, \mathrm{wR}_{2}=0.0866$ \\
\hline Final $\mathrm{R}$ indexes [all data] & $\mathrm{R}_{1}=0.0492, \mathrm{w} \mathrm{R}_{2}=0.1060$ & $\mathrm{R}_{1}=0.0488, \mathrm{wR}_{2}=0.0923$ \\
\hline Largest diff. peak/hole/ e $\AA^{-3}$ & $0.65 /-0.41$ & $0.65 /-0.42$ \\
\hline
\end{tabular}

${ }^{\mathrm{a}} \mathrm{R}_{1}=\sum\left\|\mathrm{F}_{\mathrm{o}}|-| \mathrm{F}_{\mathrm{c}}\right\| /\left[\sum\left|\mathrm{F}_{\mathrm{o}}\right|\right]{ }^{\mathrm{b}} \mathrm{wR}_{2}=\left[\sum\left[\mathrm{w}\left(\mathrm{F}_{0}^{2}-\mathrm{F}_{\mathrm{c}}^{2}\right)^{2}\right] / \sum\left[\mathrm{w}\left(\mathrm{F}_{0}^{2}\right)^{2}\right]\right]^{1 / 2} \quad \mathrm{w}=1 / \sigma^{2}\left(\mathrm{~F}_{\mathrm{o}}{ }^{2}\right)+(\mathrm{aP})^{2}+\mathrm{bP}$ where $\mathrm{P}=\left[\max \left(0\right.\right.$ or $\left.\left.\mathrm{F}_{\mathrm{o}}^{2}\right)+2\left(\mathrm{~F}_{\mathrm{c}}{ }^{2}\right)\right] / 3$

Table S2. Selected bond distances ( $\mathrm{A}$ ) and bond angles (deg) for $\mathbf{1}$ and $\mathbf{2}$.

\begin{tabular}{llll}
\hline & 1 & & 2 \\
\hline Zn1-N2 & $2.183(2)$ & Co1-N1 & $2.158(2)$ \\
Zn1-N2A & $2.183(2)$ & Co1-N1A & $2.158(2)$ \\
Zn1-N9 & $2.096(2)$ & Co1-N2 & $2.052(2)$ \\
Zn1-N9A & $2.096(2)$ & Co1-N2A & $2.052(2)$ \\
Zn1-N1A & $2.218(2)$ & Co1-N9A & $2.119(2)$ \\
Zn1-N1 & $2.218(2)$ & Co1-N9 & $2.119(2)$ \\
Zn2-N9 & $2.132(2)$ & Co2-N4A & $2.185(2)$ \\
Zn2-N9B & $2.132(2)$ & Co2-N4B & $2.185(2)$ \\
Zn2-N3 & $2.082(2)$ & Co2-N3A & $2.121(2)$ \\
Zn2-N3B & $2.082(2)$ & Co2-N3B & $2.121(2)$ \\
Zn2-N4 & $2.231(3)$ & Co2-N9 & $2.091(2)$ \\
Zn2-N4B & $2.231(3)$ & Co2-N9C & $2.091(2)$ \\
N2-N3 & $1.378(3)$ & N2-N3 & $1.377(3)$ \\
N6-N7 & $1.400(3)$ & N6-N7 & $1.397(3)$ \\
N2-Zn1-N1A & $111.08(8)$ & N2-Co1-N1A & $105.73(8)$ \\
N2-Zn1-N1 & $71.23(8)$ & N2-Co1-N1 & $74.51(8)$ \\
N2-Zn1-N9A & $94.34(9)$ & N2-Co1-N9A & $85.77(8)$ \\
N2-Zn1-N9 & $83.76(9)$ & N2-Co1-N9 & $94.04(8)$ \\
N9-Zn1-N9A & $99.14(14)$ & N1-Co1-N1A & $84.67(12)$ \\
N9A-Zn1-N1 & $94.46(9)$ & N1-Co1-N9 & $89.51(8)$ \\
N1-Zn1-N1A & $84.05(12)$ & N9-Co1-N9A & $103.84(12)$ \\
N9-Zn1-N9B & $102.20(13)$ & N4A-Co2-N4B & $83.39(11)$ \\
N9-Zn1-N4B & $90.41(11)$ & N3B-Co2-N4B & $72.42(7)$ \\
N3-Zn2-N9 & $86.79(9)$ & N3A-Co2-N4B & $111.92(8)$ \\
N3-Zn2-N9B & $95.97(9)$ & N9-Co2-N4B & $93.94(8)$ \\
N3B-Zn2-N4 & $103.59(10)$ & N9-Co2-N3A & $83.93(8)$ \\
N3-Zn2-N4 & $73.04(9)$ & N9-Co2-N3B & $92.54(8)$ \\
N4-Zn2-N4B & $84.49(18)$ & N9-Co2-N9C & $100.00(12)$ \\
\hline
\end{tabular}

Symmetry operation for 1. A: 5/4-y, 5/4-x, 5/4-z; B: 1-x, 3/2-y, z

Symmetry operation for 2. A: 1-x, 1/2-y, z; B: 3/4-y, 3/4-x, 3/4-z; C: 1/4+y, -1/4+x, 3/4-z 
Table S3. Bond Valence Sum (BVS) calculations for $\mathbf{1}$ and $\mathbf{2}$.

\begin{tabular}{ccccc}
\hline & \multicolumn{3}{c}{$\mathbf{2}$} \\
\hline & $\mathbf{Z}$ & & $\mathrm{Co}^{\mathrm{II}}$ & $\mathrm{Co}^{\mathrm{III}}$ \\
$\mathrm{Zn} 1$ & $2.08(0)$ & $\mathrm{Co} 1$ & $2.10(9)$ & $1.94(5)$ \\
$\mathrm{Zn} 2$ & $2.18(6)$ & $\mathrm{Co} 2$ & $1.98(0)$ & $1.82(6)$ \\
\hline
\end{tabular}

Table S4. Temperature dependence of the relaxation time for 2 obtained from $\chi^{\prime \prime}$ vs $T$ plots under zero and $30 \mathrm{kOe}$ dc fields.

\begin{tabular}{llll}
\hline$T / \mathrm{K}$ & Zero Field & $T / \mathrm{K}$ & $\begin{array}{l}30 \mathrm{kOe} \text { Field } \\
\tau / \mathrm{s}\end{array}$ \\
\hline 5.2 & $\tau / \mathrm{s}$ & 4.8 & $5.49 \times 10^{-3}$ \\
5.6 & $5.49 \times 10^{-3}$ & 5.2 & $1.61 \times 10^{-3}$ \\
6.0 & $1.61 \times 10^{-3}$ & 5.4 & $8.00 \times 10^{-4}$ \\
6.2 & $5.32 \times 10^{-4}$ & 5.6 & $5.32 \times 10^{-4}$ \\
6.4 & $2.66 \times 10^{-4}$ & 5.8 & $2.66 \times 10^{-4}$ \\
& $1.77 \times 10^{-4}$ & 6.0 & $1.77 \times 10^{-4}$ \\
\hline
\end{tabular}
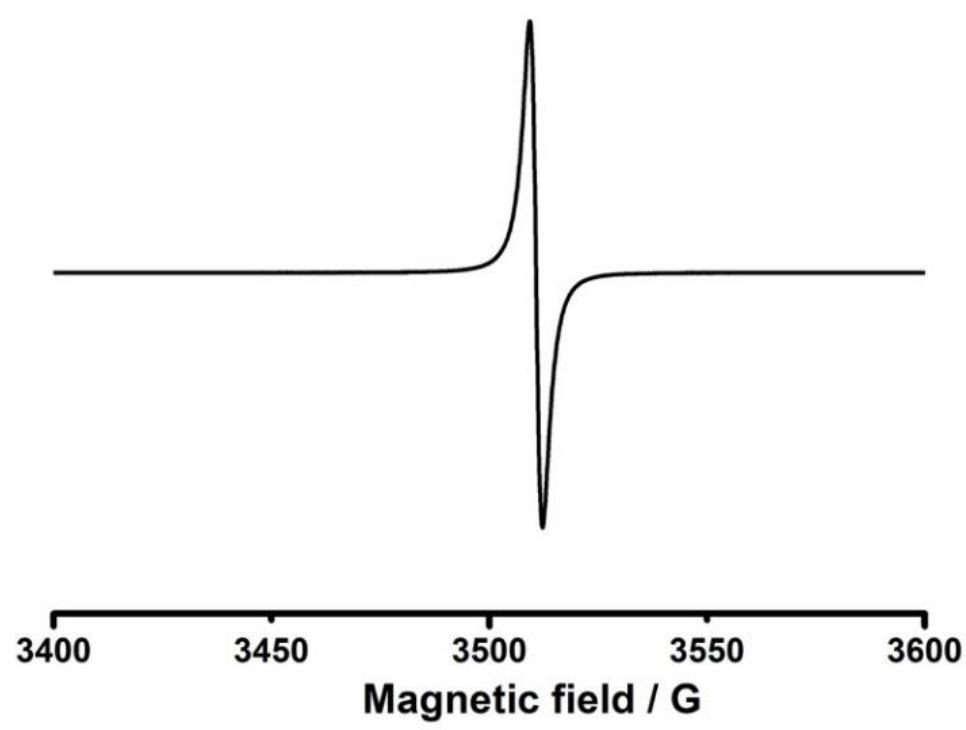

Figure S1. EPR spectrum of $\mathbf{1}$ in the solid state at room temperature giving $g_{\mathrm{rad}}=2.003$.
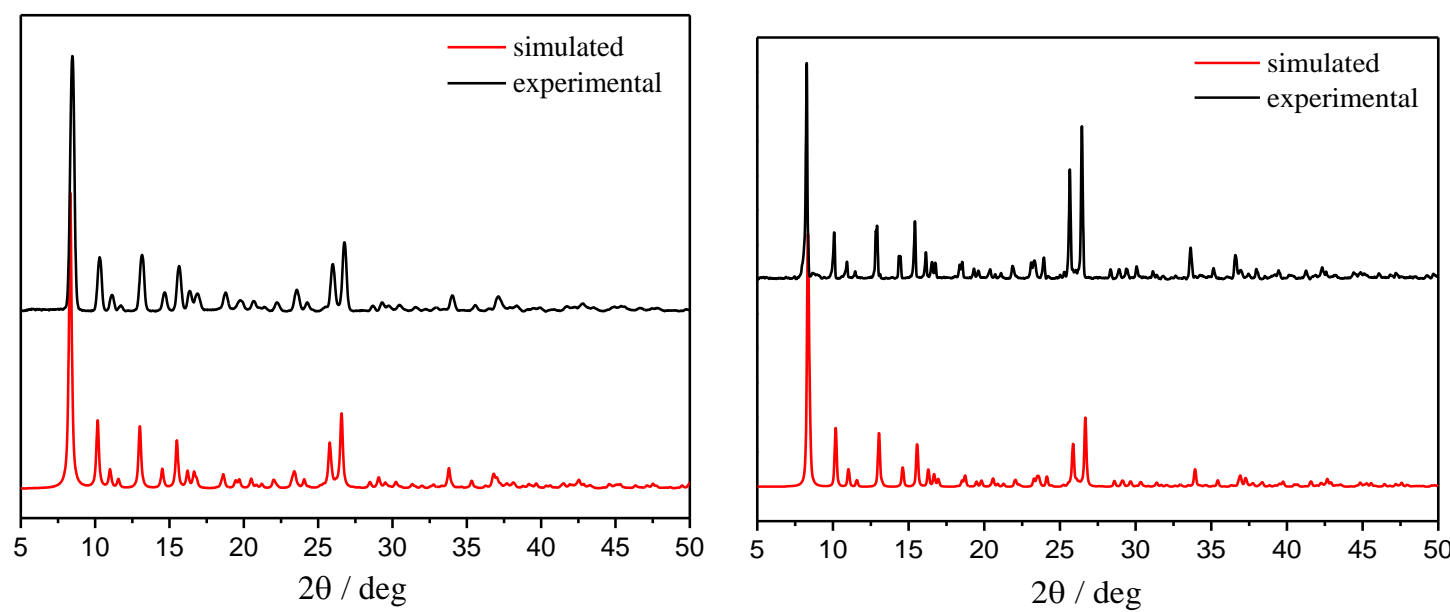

Figure S2. Powder X-ray diffraction pattern and the simulation from the single crystal data of $\mathbf{1}$ (left) and 2 (right). 

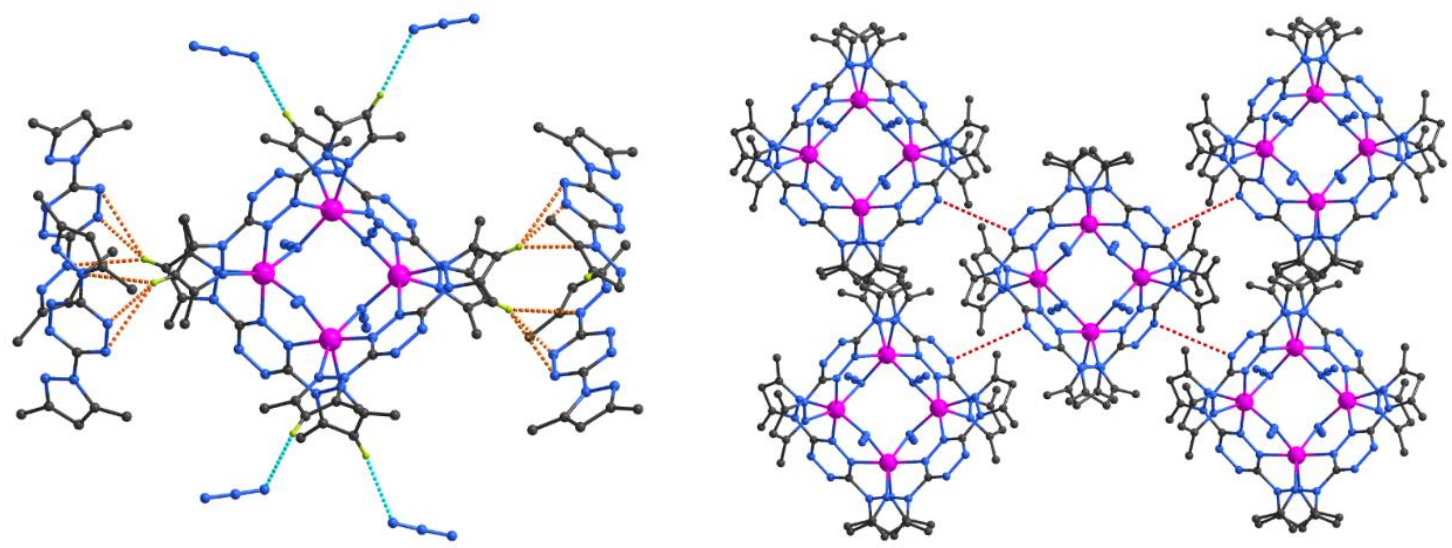

Figure S3. Ball and stick view of $\mathbf{1}$ highlighting the short contacts around the square (left) and between the packed molecules (right). The turquoise, orange and red dashed lines indicate the hydrogen bonds $(3.042 \AA), \mathrm{C} \cdots \mathrm{H} \cdots \pi$ couplings $(2.455-3.217$ $\AA$ ) and edge-to-edge $\pi \cdots \pi$ interactions $(4.107 \AA$ ), respectively. Colour codes: Zn, pink; C, grey; N, blue; H, lime. Other hydrogen or metal atoms are omitted for clarity.
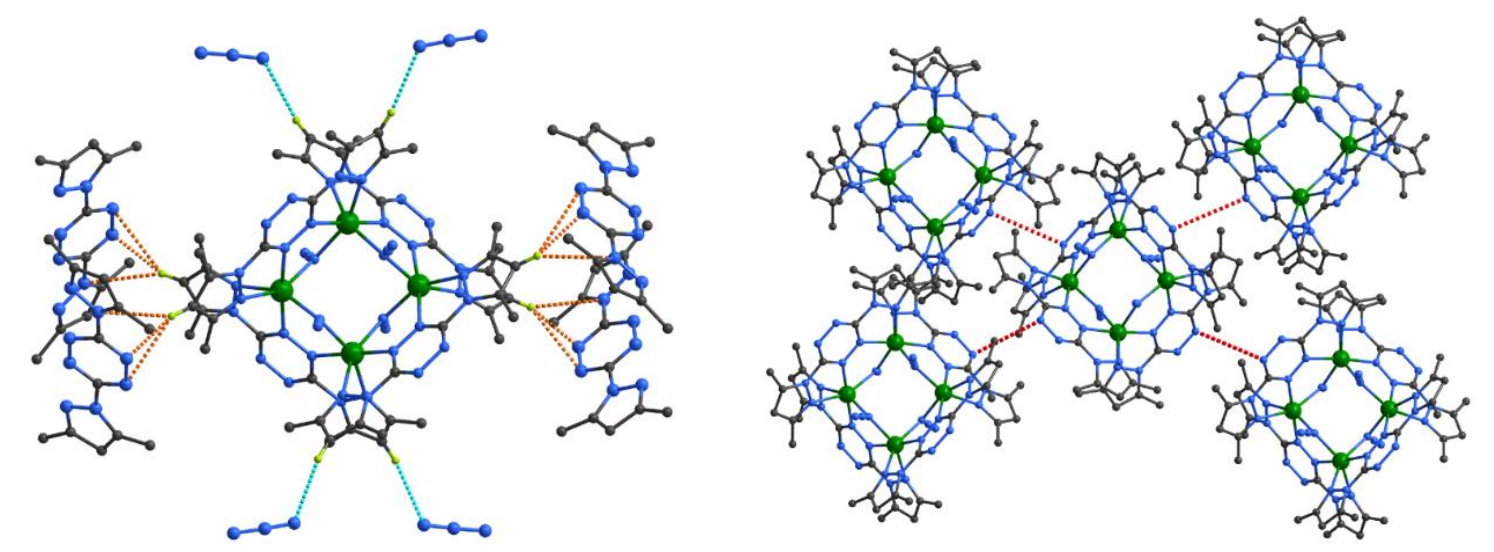

Figure S4. Ball and stick view of 2 highlighting the short contacts around the perimeter of the square (left) and between the packed molecules (right). The turquoise, orange and red dashed lines indicate the hydrogen bonds $(3.142 \AA), \mathrm{C} \cdots \mathrm{H} \cdots \pi$ couplings (2.418-3.232 A) and edge-to-edge $\pi \cdots \pi$ interactions (4.072 A). Colour codes: Co, green; C, grey; N, blue; H, lime. Other hydrogen or metal atoms are omitted for clarity.

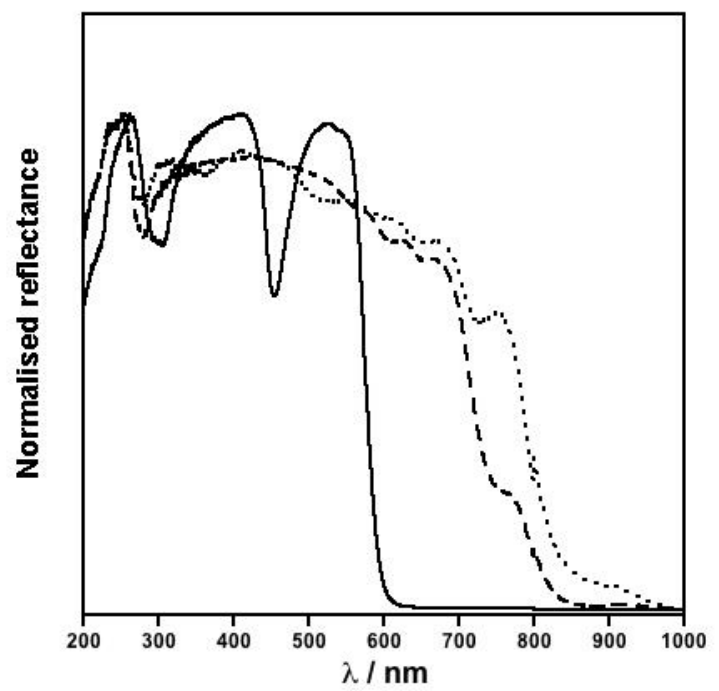

Figure S5. Solid state absorption spectra of neutral bpztz ligand (solid line), 1 (dashed line) and 2 (dotted line). 


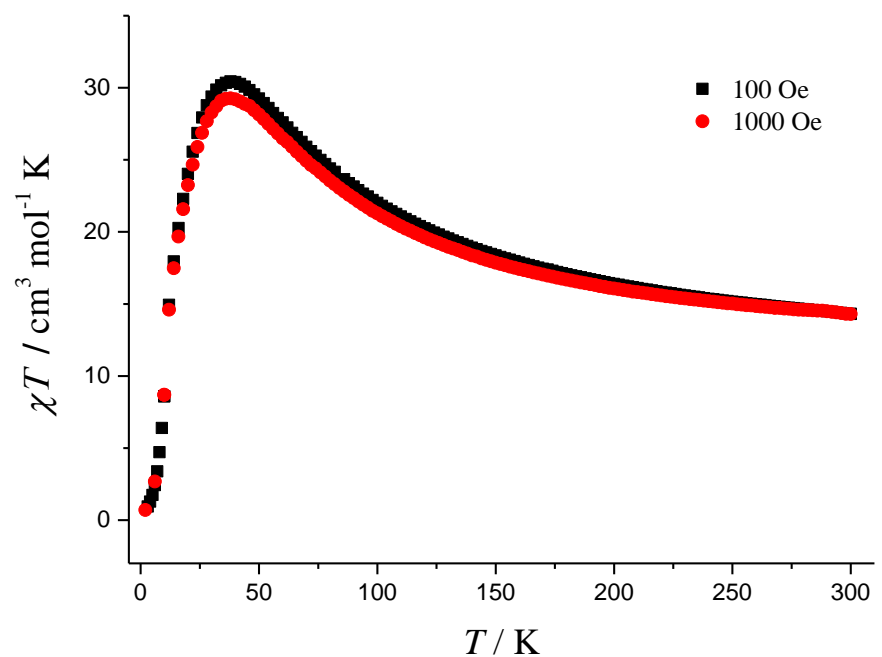

Figure S6. Variable-temperature magnetic susceptibility data for 2 under 100 Oe and 1 kOe dc fields.

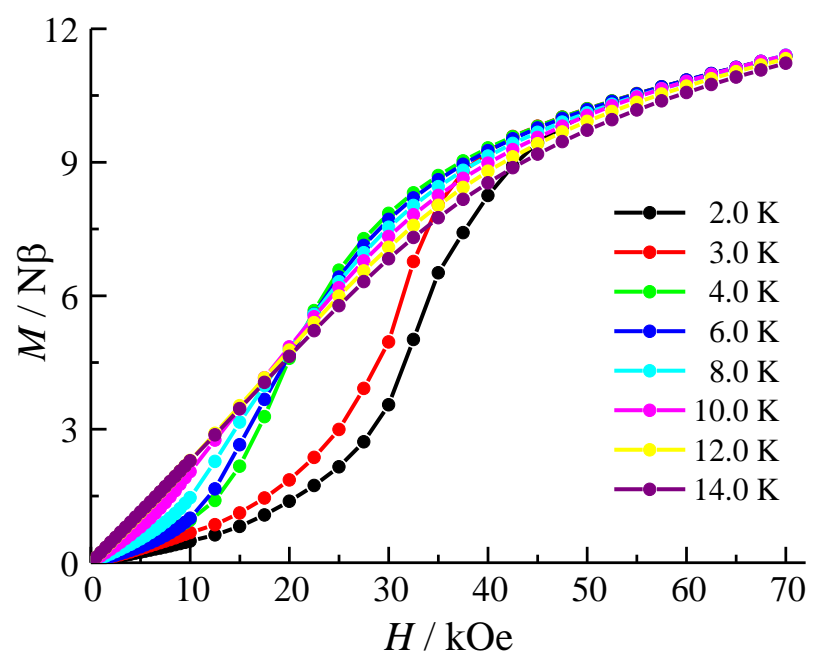

Figure S7. Field dependence of the magnetization for 2 between 2 and $14 \mathrm{~K}$. Solid lines are guides for the eye.

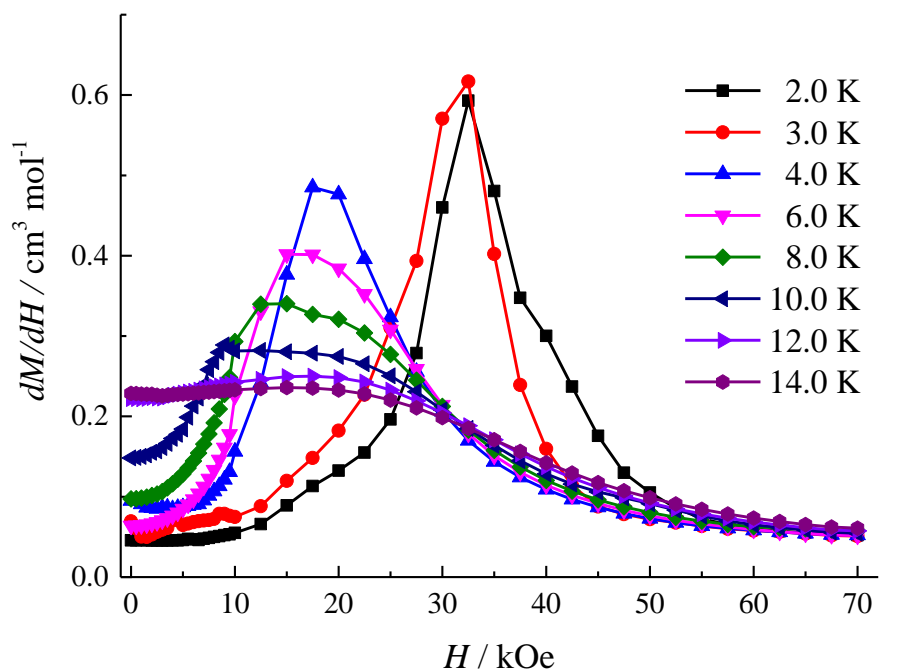

Figure S8. First field derivative of the magnetization as a function of the applied dc field for 2 between 2 and 14 K. Solid lines are guides for the eye. 


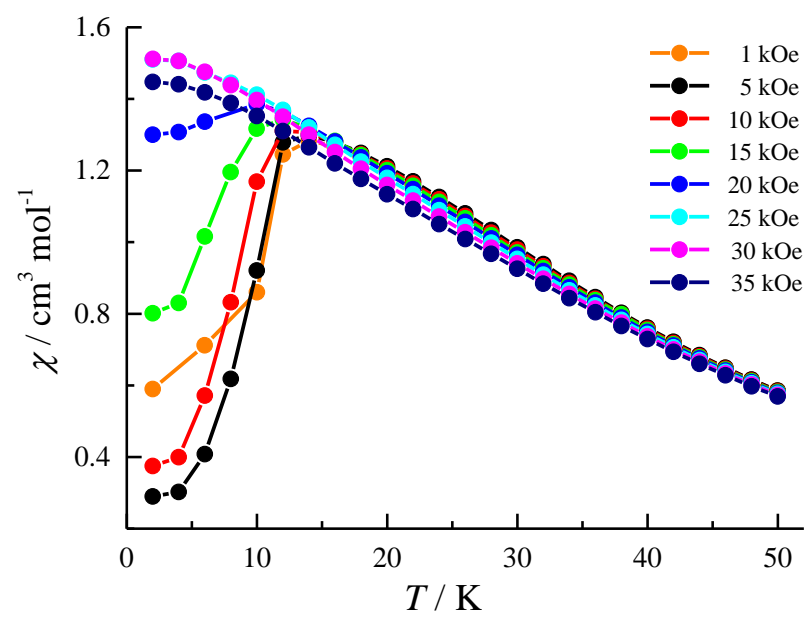

Figure S9. Temperature dependence of the magnetic susceptibility for $\mathbf{2}$ as a function of applied dc fields. Solid lines are guides for the eye.

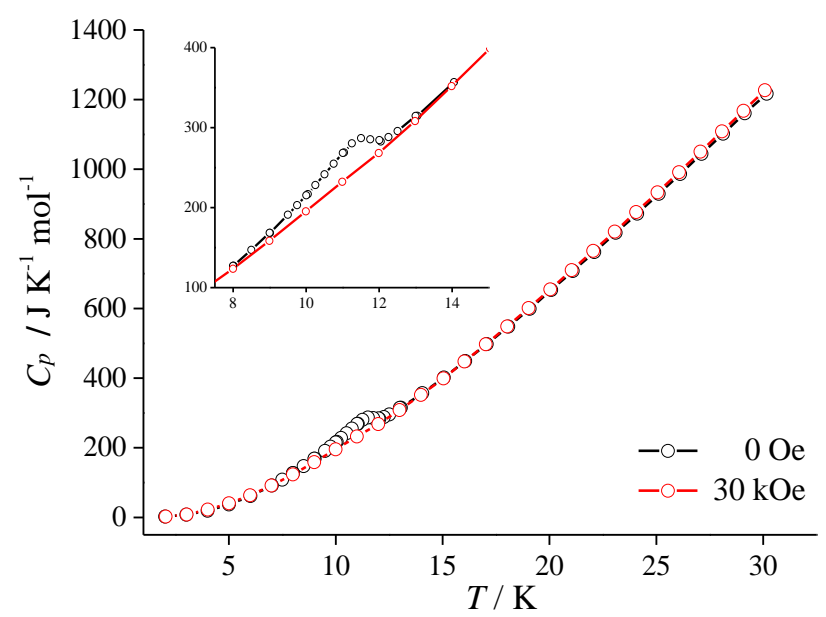

Figure S10. Variable temperature heat-capacity plots of 2 under zero and $30 \mathrm{kOe}$ dc fields. Inset shows the enlarged plots over the temperature range of 8-14 K. Solid lines are guides for the eye.
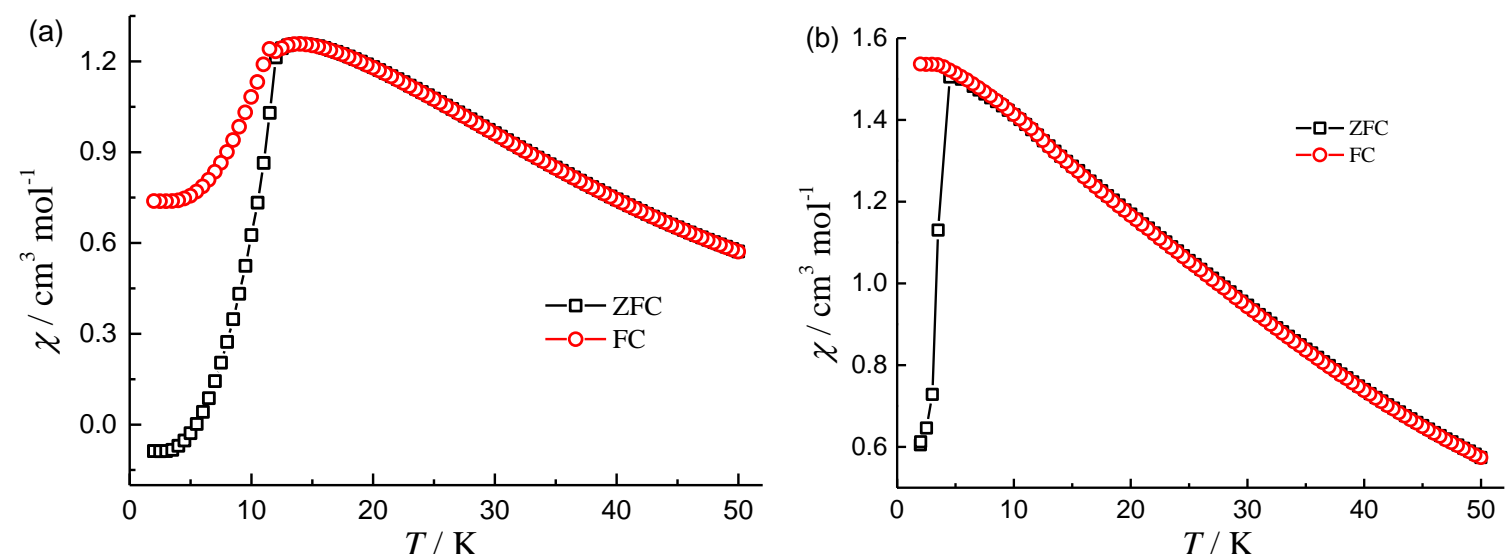

Figure S11. ZFC and FC magnetization data for 2 measured with an applied dc field of 50 Oe (a) and $30 \mathrm{kOe}$ (b). Solid lines are guides for the eye. 


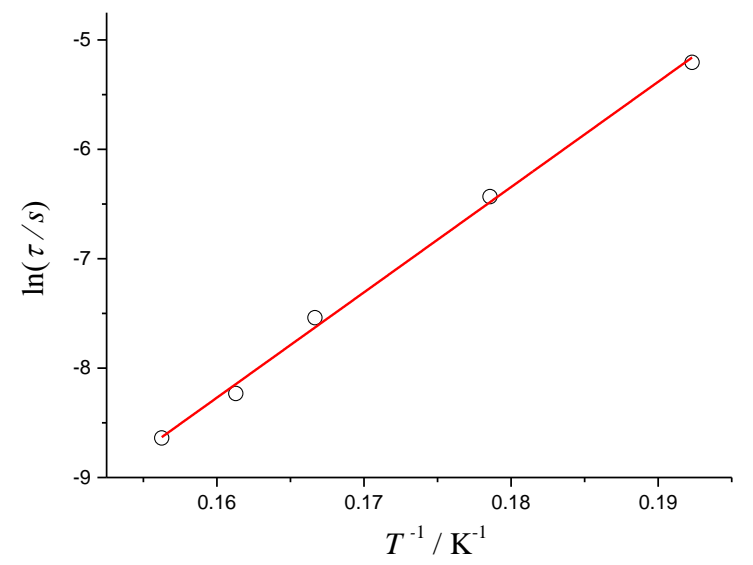

Figure S12. Arrhenius plots of 2 at zero dc field. The solid line represents the fit to the data.

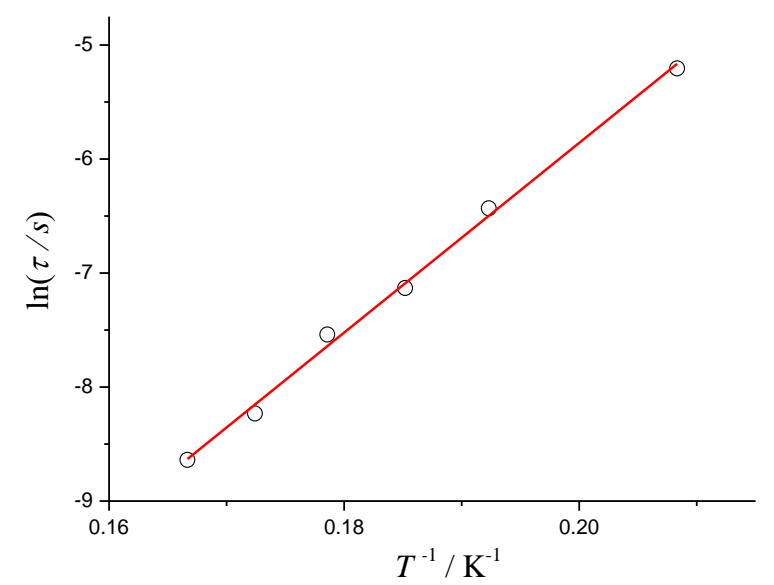

Figure S13. Arrhenius plots of $\mathbf{2}$ at $30 \mathrm{kOe}$ applied dc field. The solid line represents the fit to the data.

\section{References}

1 (a) G. M. Sheldrick, SADABS, v.2.01, Bruker/Siemens Area Detector Absorption Correction Program. Bruker AXS:

Madison, Wisconism, 1998; (b) G. M. Sheldrick, SHELXL-2014, Program for Crystal Structure Refinement. University of Göttingen, Göttingen, Germany, 2014; (c) G. M. Sheldrick, SHELXL-2014, Program for the solution of crystal structures. University of Göttingen, Göttingen, Germany, 2014; Vol. 8.

2 G. A. Bain and J. F. Berry, Diamagnetic Corrections and Pascal's Constants. J. Chem. Educ., 2008, 85, 532. 